\title{
PRELIMINARY CLINICAL RESULTS OF IMPLANTATION OF BIODEGRADABLE PERICARDIAL SUBSTITUTE IN PEDIATRIC OPEN HEART OPERATIONS
}

\author{
Afksendiyos Kalangos, MD, PhD, and Bernard Faidutti, MD, Geneva, Switzerland
}

An increasing number of repeat operations done for various reasons has forced surgeons to face the problem of pericardial adhesions that could place some sites of the heart at risk during repeat sternotomy. Therefore various pericardial substitutes have been developed and tested with long-term clinical outcomes not well known. Recently, a new concept based on the regenerative potential of pericardial mesothelium onto a biodegradable patch has been the focus of great enthusiasm after the experimental study done by Malm and associates. ${ }^{1,2}$ In their study, the effects of the biodegradable polyhydroxybutyrate (PHB) patch on adhesion formation, preservation of the coronary anatomy, and pericardial regeneration were investigated. In animals treated with $\mathrm{PHB}$ patches, the adhesion formation was minimized and the visibility of coronary anatomy was preserved significantly as a result of the demonstrated fact that the patch could act as a scaffold for regeneration of mesothelium-like cells with preserved prostacyclin activity. ${ }^{1}$ However, since the clinical application of this patch (provided by Astra Meditec, Mölndal, Sweden) was first done, no evaluation for adhesion development and epicardial reaction to this patch in human beings has been reported, certainly because of a limited number of cases of reoperation in which the patch was implanted.

Since 1992 the biodegradable PHB patch was used in our institution as a pericardial substitute in 65 pediatric cases that required open heart operations because of various congenital lesions. In all these cases the anterior autologous pericardium was resected in order to be used either in the reconstruction of the right ventricular (RV) outflow tract or in the closure of ventricular septal defects. The PHB patch was applied before sternal closure at the site of the harvested pericardium by interrupted 5-0 monofilament stitches to the edge of the native pericardium. Two drains were placed into the mediastinum after the patch was spread as smoothly as possible over the anterior and inferior surfaces of the RV, ascending aorta, and part of the pulmonary artery.

To date, five children have required reoperation because of various reasons: residual or recurrent $\mathrm{RV}$ outflow

From the University Cantonal Hospital of Geneva, Clinic for Cardiovascular Surgery, Geneva, Switzerland.

Received for publication April 8, 1996; accepted for publication May 16, 1996.

Address for reprints: Afksendiyos Kalangos, $\mathrm{MD}, \mathrm{PhD}$, University Cantonal Hospital of Geneva, Clinic for Cardiovascular Surgery, 24, rue Micheli-du-Crest, 1211 Geneva 14, Switzerland.

J Thorac Cardiovase Surg 1996;112:1401-2

Copyright 1996 by Mosby-Year Book, Inc.

$0022-5223 / 96 \$ 5.00+0 \quad \mathbf{1 2 / 5 4 / 7 5 2 0 7}$ tract stenosis in three cases, residual ventricular septal defect in one case, and RV aneurysm formation in one case. The mean interval between the two operations was 186.4 days (range 76 to 471 days). No excessive postoperative bleeding or infections were noted after the first operation. Four children who underwent reoperation after a mean duration of $\mathbf{1 1 5 . 2}$ days (range 76 to 154 days) were evaluated for adhesion formation and visibility of coronary anatomy. In these cases at the time of reoperation, adhesions between the sternum and PHB patch and between the area of the patch and epicardium were graded as moderate. Adhesions between native pericardium and epicardium were also evaluated as moderate except in one case in which the native pericardium was severely adhered to a previously implanted aortic homograft. In these four cases, the visibility of the coronary anatomy was blurred in both epicardial areas in contact with the PHB patch and native pericardium. At the time of reoperation, the patch had an aspect of a fibrinous capsule covering the epicardial surface that allowed the underlying anatomy to be distinguished during the dissection, which did not result in bleeding. There was not a clear demarcation at the level of the suture line between patch and native pericardium. No histologic specimens of the patch or epicardium were obtained.

In the last case, that of a 2-year-old child who had initially undergone correction of tetralogy of Fallot with an aortic homograft implantation between the RV and pulmonary artery bifurcation, a significant stenosis developed at the site of anastomosis between the RV and homograft 14 months after operation. At the time of reoperation 16 months after operation, no adhesions between the sternum and anterior RV epicardium were noted after resternotomy. The PHB patch was completely absorbed and the underlying anatomy at the site of patch implantation was clearly recognizable. On the contrary, severe adhesions between native pericardium and underlying heart structures and especially the homograft necessitated a sharp dissection that resulted in bleeding.

Our preliminary clinical results suggest that the PHB patch was well tolerated and remained basically as a fibrinous sac maintaining an acceptable plane of cleavage between the patch and epicardium in the first 5 months after operation. Absorption of the patch probably occurs more rapidly in human beings than was observed in animals. ${ }^{1}$ Of course, this time-related phenomenon is also related to the severity of the early postoperative inflammatory reaction, which will be more pronounced in cases with anatomic and biologic structural tissue modifications caused by several cardiac incisions or suture lines with the use of cardiopulmonary bypass. In the first few months after operation, the severity of the inflammatory reaction and number of macrophages will regress proportionally to the absorbed amount of polymer and to increased func- 
tional properties of the regenerated mesothelium-like cells that could better stimulate and facilitate the absorption. We believe that adhesion formation and functional activity of the regenerated tissue onto the PHB patch should be reevaluated in an experimental study under conditions resembling those of open heart operations in human beings. A more detailed analysis of the interaction between the biodegradable patch and epicardium should be considered with the enhanced inflammatory reaction induced by cardiopulmonary bypass and surgical trauma. Moreover, why could one not create biodegradable patches soaked in some antiinflammatory substances such as protease inhibitors that could attenuate postbypass inflammatory reactions?

On the basis of our preliminary clinical experience, the biodegradable patch seems to be an interesting material as a pericardial substitute in patients who might require reoperation in the medium or long term when primary pericardial closure is not possible.

\section{REFERENCES}

1. Malm T, Bowald S, Bylock A, Busch C. Prevention of postoperative pericardial adhesions by closure of the pericardium with absorbable polymer patches. J Thorac Cardiovasc Surg 1992;104:600-7.

2. Malm T, Bowald S, Bylock A, Saldeen T, Busch C. Regeneration of pericardial tissue on absorbable polymer patches implanted into the pericardial sac. Scand J Thorac Cardiovasc Surg 1992;26:15-21.

\section{STERNAL HEMANGIOMA: A RARE TUMOR}

Benjamin Medalion, MD, ${ }^{a}$ Ilan Bar, MD, ${ }^{a}$ Rami Neuman, MD, ${ }^{\mathrm{b}}$ Yaron Shargal, MD, ${ }^{\mathrm{a}}$ and Gideon Merin, MD, Jerusalem, Israel

Primary tumors of the bony chest wall are uncommon. ${ }^{1}$ Among those tumors, sternal tumors are extremely rare ${ }^{2}$ and usually malignant. ${ }^{2,3}$ In this case report, we discuss a benign hemangioma of the sternum in a 30 -year-old woman who had a 2-year history of chest pain without objective physical findings. She underwent a bone scan, with results regarded as normal. Computed tomography demonstrated a round mass in the body of the sternum (Fig. 1, $A$ ). On magnetic resonance imaging (Fig. 1, B), the mass was found to be located in the lower part of the sternal body to invade the sternal cortex. An open biopsy sampling of the mass was performed elsewhere, and a benign hemangioma was diagnosed. During the biopsy procedure, the patient had extensive bleeding, which was finally controlled.

Subsequent magnetic resonance imaging 2 months later demonstrated an enlargement of the mass, and she had an elective operation. The left and right internal thoracic arteries were divided at the manubrium. Each costochondral junction was divided on both sides after the intercostal vessels had been ligated and divided. The sternum was transected transversely through the second intercostal space; the resected sternum and the mass were then separated from the mediastinum and removed. The pec-

From the Departments of Thoracic and Cardiovascular Surgery ${ }^{\mathrm{a}}$ and Plastic Surgery, ${ }^{\mathbf{b}}$ Hadassah University Hospital, Jerusalem.

Received for publication Jan. 22, 1996; accepted for publication Feb. 8, 1996.

J Thorac Cardiovasc Surg 1996;112:1402-3

Copyright (C) 1996 by Mosby-Year Book, Inc.

$0022-5223 / 96 \$ 5.00+0 \quad \mathbf{1 2 / 5 4 / 7 2 6 6 1}$ toralis major muscles were mobilized and used as in situ flaps to close the wound.

The patient had uneventful operative and postoperative courses and went home 8 days after the operation. Histologic examination of the mass revealed an encapsulated $5 \times 5 \times 9 \mathrm{~cm}$ hemangioma that replaced almost the entire resected sternum. At 6-month follow-up the patient had regained a normal lifestyle, with no chest pain and no signs of recurrence.

A search of the literature revealed only one similar case, that of a 38-year-old woman who reported pain in the midanterior area of the chest. ${ }^{4}$ This patient had a history of similar intermittent pain for 15 years. Physical examination revealed only slight prominence and tenderness of the superior aspect of the sternal body. The patient underwent a surgical excision of the sternal lesion.

Benign sternal tumors are extremely rare, and all sternal tumors should be considered malignant until proved otherwise. ${ }^{4}$ Hemangiomas of the chest wall are most often encountered in the vertebra, ribs, and skeletal muscles. ${ }^{5}$ Although hemangiomas are quite common tumors, no hemangiomas of the sternum have been described except for the case published by Boker and coworkers ${ }^{4}$ and the case described here by us. Irradiation therapy, interferon therapy, and selective embolization of feeding vessels have been suggested as alternatives to surgical treatment, especially for children and patients with hemangiomas of the skin. ${ }^{6-8}$ Because there is no sharp distinction between benign hemangiomas and lowgrade malignant hemangioendotheliomas and because of the relatively high recurrence rate of hemangiomas, ${ }^{5}$ we decided to operate on this patient and to resect the tumor with adjacent sternal tissue. 